\title{
Téoros
}

Revue de recherche en tourisme

\section{L’accueil : une activité humaine}

\section{Michel G. Langlois}

Volume 10, numéro 2, juillet 1991

Accueil et tourisme

URI : https://id.erudit.org/iderudit/1078953ar

DOI : https://doi.org/10.7202/1078953ar

Aller au sommaire du numéro

Éditeur(s)

Université du Québec à Montréal

ISSN

0712-8657 (imprimé)

1923-2705 (numérique)

Découvrir la revue

Citer ce document

Langlois, M. G. (1991). L’accueil : une activité humaine. Téoros, 10(2), 2-2.

https://doi.org/10.7202/1078953ar d'utilisation que vous pouvez consulter en ligne.

https://apropos.erudit.org/fr/usagers/politique-dutilisation/ 
Présentation

Michel G. Langlois

\section{L'accueil:}

\section{une activité humaine}

Un bon accueil n'est pas l'effet du hasard.

Accueillir a etté de tous les temps une activité importante et significative chez les gens tant à la période des Croisades qu'aujourd'hui. Tantôt pour se déculpabiliser, tantôt pour inconsciemment se garantir la réciprocité. L'accueil est un événement.

Quelle est sa dynamique? Qui doit en assumer la responsabilité? Jusqu'à quel point sa qualité influence l'image d'une entreprise, d'une ville, d'un Etat? Le présent numero de Téoros parcourtces divers aspects de l'accueil.

Un survol de l'histoire nous enseigne que l'homme a toujours été préoccupé par la venue d'étrangers dans sa communaute. Cet accueil qui variera avecles moeurs etles cultures n'en est pas moins chargé d'intensité variée. II ne laisse certainement personne indifférent.

On ne peut séparer accueil et hospitalite. Peu importe la qualite de l'accueil, il aura certes un impact sur la perception de l'hospitalité que le visiteur évaluera par une expérience positive ou négative.

On ne peut fuir l'accueil et l'hospitalité. IIs seront là. La question n'est pas d'éviter ou de saisir. Dans la perspective du visiteur, il y aura toujours un jugement, une opinion sur l'accueil qu'il recevra. Qu'il soit planifié, adapté ou non, l'effet sera présent et marquant.

Il revient donc aux organismes responsables de l'accueil de faire en sorte qu'il soit bien géré et conforme aux attentes des clients visiteurs.

Mais à qui la responsabilité? L'État doit-il être le moteur ou laisser les organismes réceptifs à leur bon gré? ? L'État doitil influencer les joueurs? Doit-il former, inciter et supporter les agents d'accueil ? Qui est responsable du positionnement d'hospitalité d'une région touristique? L'Etat ou les divers intervenants touristiques privés ou les deux et dans quelle mesure?

I/ nous apparait essentiel que, pour présenter une image cohérente et un positionnement commercial valable, l'État doit assumer un certain leadership au niveau de la diffusion en fournissant non seulement le support au niveau de fimage mais egalementen fournissant un support technique d'accẻs à l'information pour les visiteurs potentiels.

Mais qui fait limage d"accueil ? Est-ce l'État ou les prestataires de services. L'État ne peut-il que véhiculer ce qui existe.

La communication client est-elle chose simple? Peut-on tout englober dans une simple image? Peut-on offrir tout a tout le monde et rendre tout le monde heureux? Chaque clientèle nécessite sa structure d'accueil et d'hospitalité. L'expérience attendue variera avec le type de clients.

Une bonne communication client suppose que l'on aura bien segmenté son marché; que l'on aura ciblé son action et que notre structure d'accueil répondra aux besoins précis de la clientèle visée. Notre image dépend non seulement de la communication préalable au deploiement mais de la communication pendant l'expérience d'accueil que le client devra vivre. C'est cette expérience qu'il comparera aux promesses qu'on luia faites etauxattentes qu'il aura. Pour cette raison, il est primordial de bien elaborer notre concept d'offre aux clients en le basant sur des attentes précises et non banales.

On ne peut pas éviter en traitant de l'accueil de discuter de la relation visiteurs-visités en la plaçant dans une perspective de processus interactif.

C'est à ce niveau que nous retrouvons le coeur de l'accueil et de l'hospitalité. L'accueil est une activité humaine qui existe dans la mesure où deux être humains communiquent $d^{\prime} u n e$ façon inductive ou verbale. A cet effet le véhicule Hôte ou la personne qui accueille a une responsabilité importante et transmet, a chaque instant de contact, des eléments qui permettent aux visiteurs de juger.

Si nous voulons modifier la qualité de l'accueil, c'est au niveau des individus qui font cet accueil que nous devons intervenir. En effet, s'il y a dans toute communication la relation et le contenu, la relation qui est, elle, le véhicule du contenu semble occuper une position privilégiée.

Les facteurs aussi elémentaires que l'empathie et le soin de la tenue peuvent influencer positivement ou négativement l'opinion du visiteur.

Le produit d'accueil est en grande partie un produit d'attitude, de disponibilité; il sous-tend la notion orientale de service dans laquelle servir est un honneur et non pas un emploi de deuxieme ordre.

Modifier ou façonner l'attitude du personnel d'accueil est en soi un programme important. Mais où est la solution ? Est-ce une question de formation ou une question de supervision?

Les programmes de formation ne peuvent fournir que des techniques ou des movens de bien faire les choses. Cependant, ils n'interviennent pas au niveau de la motivation qui peut influencer l'attitude.

La qualité de l'accueil, dans sa perspective dynamique, est essentiellement un probleme de gestion. L'attitude du personnel d'accueil est sans doute conditionnée par la relation patron-employés.

Même si nous traitons de l'accueil dans ses dimensions plus larges, la clef d'un accueil façonné a la clientele des visiteurs est conditionnée par la qualite de la supervision du personnel en contact avec les clients. Si cette relation de basen'est pas réalisée dans une perspective de valorisation du visiteur, la notion même d'accueil aura failli au coeur de la définition de l'hospitalité : une attitude chaleureuse, empathique et serviable. 